\title{
Reverse Phase Protein Array: A Tool for Signaling Pathway Profiling in the Era of Genome Resequencing
}

\author{
Tesshi Yamada
}

Published online: 5 March 2014

(c) Springer Science+Business Media New York 2014

Current molecular-targeted cancer therapeutics are designed to target the dysregulation of intracellular signaling that ensues from the accumulation of genetic and epigenetic alterations that exemplify cancer. Since many intracellular signaling pathways rely primarily on protein phosphorylation, comprehensive profiling of protein phosphorylation is essential not only for understanding the molecular mechanisms that underlie the occurrence and progression of cancer but also for the development of new preventive, diagnostic, and therapeutic methods. Gel electrophoresis- and mass spectrometry-based proteomics technologies have rapidly advanced in recent years, enabling the quantitation of protein expression in clinical samples, although the sensitivity of these technologies is insufficient for the comprehensive profiling of low-abundance signaling proteins.

Reverse-phase protein array or reverse phase protein microarray (RPPA or RPMA) [1] is a highly miniaturized dot-blot platform that enables the simultaneous quantification of protein expression in a large number of biological samples. As many as 6,144 protein samples can be spotted onto standard glass slides using innovative pin spotting technology [2]. RPPA is unsurpassed by other proteomics technologies regarding its sensitivity; furthermore, only nano- or picoliter-level protein samples are required. Since the supply of clinical materials is often limited, RPPA, due to its high sensitivity, is highly advantageous in clinical proteomics. The RPPA platform, recently adapted to the Cancer Genome Atlas (TCGA) Network [3], will be incorporated into clinical trials as a method for monitoring

T. Yamada $(\bowtie)$

National Cancer Center Research Institute, 5-1-1 Tsukiji,

Chuo-ku, Tokyo 104-0045, Japan

e-mail: tyamada@ncc.go.jp molecular responses to molecular-targeted therapeutics in small biopsy samples. We have been using RPPA as a validation method to determine the clinical utility of candidate serum/plasma biomarker proteins that have been discovered using other proteomics technologies [4]. As an example, Byers et al. [5] identified poly(ADP-ribose) polymerase-1 (PARP-1) as a potential therapeutic target for small-cell lung cancer using RPPA.

In this issue of Digestive Diseases and Sciences, Huang et al. [6] at the University of Texas MD Anderson Cancer Center describe their search for proteins that are differentially expressed between pancreatic cancer and normalappearing tissues of 15 patients using a panel of 130 antibodies and RPPA. Among the four proteins that were selected under strict statistical criteria, they were able to confirm the increased expression of three proteins in pancreatic cancer via immunoblotting: AKT, $\beta$-catenin, and GRB2-associated-binding protein 2 (GAB2). Among these, $\mathrm{AKT}$ is an oncogenic serine/threonine kinase that functions downstream of the phosphatidylinositol-3 kinase (PI3K)/ phosphatase and tensin homolog deleted from the chromosome 10 (PTEN) pathway. $\beta$-Catenin is an oncogenic effector of the Wnt signaling pathway, and GAB2 is a signaling protein that serves as a downstream adaptor for membrane receptors. The involvement of GAB2 was not described previously in the literature, its discovery made possible only through the current proteomics approach.

Nevertheless, the present study also had some limitations. It was necessary to microdissect ductal epithelial cells in order to identify the alterations that occur during carcinogenesis, a laborious procedure. Furthermore, the current findings are based on a small population and thus must be replicated in an independent patient cohort. Future functional studies will also be necessary to determine the biological significance of the reported findings. 


\section{References}

1. Nishizuka S, Charboneau L, Young L, et al. Proteomic profiling of the NCI-60 cancer cell lines using new high-density reverse-phase lysate microarrays. Proc Natl Acad Sci USA. 2003;100: 14229-14234.

2. Murakoshi Y, Honda K, Sasazuki S, et al. Plasma biomarker discovery and validation for colorectal cancer by quantitative shotgun mass spectrometry and protein microarray. Cancer Sci. 2011;102:630-638.

3. Cancer Genome Atlas Network. Comprehensive molecular portraits of human breast tumours. Nature. 2012;490:61-70.
4. Matsubara J, Honda K, Ono M, et al. Identification of adipophilin as a potential plasma biomarker for colorectal cancer using labelfree quantitative mass spectrometry and protein microarray. Cancer Epidemiol Biomarkers Prev. 2011;20:2195-2203.

5. Byers LA, Wang J, Nilsson MB, et al. Proteomic profiling identifies dysregulated pathways in small cell lung cancer and novel therapeutic targets including PARP1. Cancer Discov. 2012;2:798-811.

6. Huang Y-J, Frazier ML, Zhang N, Liu Q, Wei C. Reverse-phase protein array analysis to identify biomarker proteins in human pancreatic cancer. Dig Dis Sci. (Epub ahead of print). doi:10.1007/ s10620-013-2938-9. 